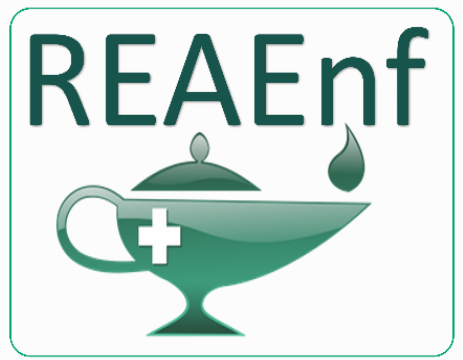

Revista Eletrônica Acervo Enfermagem
RELATO DE EXPERIÊNCIA

Recebido em: 7/2020

Aceito em: 8/2020

Publicado em: 9/2020

\title{
A gamificação como ferramenta lúdica no processo de ensino e aprendizagem na enfermagem: um relato de experiência
}

\author{
Gamification as a playing tool in the nursing teaching and learning process: an experience \\ report

\section{Gamificación como herramienta de juego en el proceso de enseñanza y aprendizaje de enfermería: un informe de experiencia}

Francisco Railson Bispo de Barros ${ }^{1 *}$, Andreia Ribeiro da Silva ${ }^{1}$, Bárbara Heleodora Siqueira Rodrigues ${ }^{1}$, Izabel Gomes da Silva1', Paulo Afonso Freitas Battanoli¹, Priscila Brito Albuquerque de Oliveira', Regina Corrêa de Souza'1.

\begin{abstract}
Resumo: Esse artigo buscou descrever a experiência da gamificação como ferramenta metodológica complementar, na perspectiva do apoio didático do processo de ensino e aprendizagem de graduandos em Enfermagem no contexto da disciplina de Saúde da Mulher. Trata-se de um estudo descritivo, tipo relato de experiência, onde foi desenvolvido um jogo em hipermídia numa sequência didática, onde se buscou a promoção do aluno no papel de protagonista de sua aprendizagem. Por meio da sequência didática e diálogo com os alunos, identificou-se que todos aderiram a atividade, participando ativamente e com entusiasmo na resolução das questões e evolução do jogo, indicando uma ótima aceitação dos métodos utilizados. Pode-se observar a adequação da gamificação como ferramenta metodológica de apoio no processo de ensino e aprendizagem, proporcionando fixação do conhecimento científico da temática abordada. Com isso, conclui-se que a gamificação pode se tornar uma relevante ferramenta didática de apoio para ensino de diversos temas à graduandos de Enfermagem.
\end{abstract}

Palavras-chave: Ensino, Apoio didático, Educação em enfermagem.

\begin{abstract}
This article sought to describe the experience of gamification as a complementary methodological tool, in the perspective of the didactic support of the teaching and learning process of undergraduate nursing students in the context of the Women's Health discipline. It is a descriptive study, like an experience report, in which a hypermedia game was developed in a didactic sequence, in which the promotion of the student in the role of protagonist of his learning was sought. Through the didactic sequence and dialogue with the students, it was identified that everyone joined the activity, participating actively and enthusiastically in solving the questions and evolving the game, indicating an excellent acceptance of the methods used. It is possible to observe the adequacy of gamification as a methodological support tool in the teaching and learning process, providing fixation of scientific knowledge of the subject addressed. With that, it is concluded that gamification can become a relevant didactic support tool for teaching several subjects to nursing students.
\end{abstract}

Keywords: Teaching, Didactic support, Nursing education.

${ }^{1}$ Centro Universitário do Norte (UNINORTE). Manaus - AM. *E-mail: enf.franciscobarros@gmail.com 
Resumen: Este artículo buscó describir la experiencia de la gamificación como herramienta metodológica complementaria, en la perspectiva del soporte didáctico del proceso de enseñanza y aprendizaje de estudiantes de pregrado de enfermería en el contexto de la disciplina Salud de la Mujer. Es un estudio descriptivo, tipo de informe de experiencia, en el que se desarrolló un juego hipermedia en una secuencia didáctica, en el que se buscó la promoción del alumno en el papel de protagonista de su aprendizaje. A través de la secuencia didáctica y el diálogo con los estudiantes, se identificó que todos se unieron a la actividad, participando activamente y con entusiasmo en la resolución de los problemas y la evolución del juego, lo que indica una excelente aceptación de los métodos utilizados. Es posible observar la idoneidad de la gamificación como herramienta de apoyo metodológico en el proceso de enseñanza y aprendizaje, proporcionando una fijación del conocimiento científico del tema abordado. Con eso, se concluye que la gamificación puede convertirse en una herramienta de apoyo didáctico relevante para enseñar varias materias a estudiantes de enfermería.

Palabras clave: Docencia, Apoyo didáctico, Educación en enfermería.

\section{INTRODUÇÃO}

O mundo cada vez mais dinâmico aumentou as formas de produção e obtenção de saberes, onde o processo do ensino e aprendizagem vem sofrendo profundas adaptações com o passar do tempo. Muitos dos professores, facilitadores e mediadores desse processo, ainda seguem estagnados a paradigmas considerados ultrapassados, utilizando-se de métodos tradicionais, aulas expositivas enfadonhas e pouco produtivas (AYRES MAC, et al., 2016).

Nesse contexto, tem-se observado um ascendente movimento de reordenamento dos currículos superiores da saúde, assim como a busca por implementação de metodologias ativas de ensino e aprendizagem, centradas no aluno e desvinculando à percepção de soberania do conhecimento focada no facilitador e mediador desse processo, o professor (FABBRO MRC, et al., 2018).

Atualmente, as Diretrizes Curriculares Nacionais (DCN's) do curso de graduação em Enfermagem corrobora esta ideia, uma vez que reconhece o graduando como sujeito do processo de formação, utilizando metodologias ativas de ensino e aprendizagem e abordagens inovadoras que estimulem nos sujeitos participantes a aprendizagem significativa, como o uso das múltiplas tecnologias em favor desse processo (BRASIL, 2018).

Partindo dessa abordagem inovadora das metodologias ativas e do uso das múltiplas tecnologias, destacamos a gamificação como um desses recursos pedagógicos que pode promover o desenvolvimento socioafetivo, psicomotor, linguístico, moral e cognitivo do graduando em enfermagem. A gamificação se utiliza de elementos de jogos em contextos de não jogos, possibilitando uma experiência diferente que supra às expectativas e motivações do aluno (KAPP KM, 2012).

Entende-se que a gamificação é o processo de ensino e a aprendizagem com base em jogos, constituem-se em uma abordagem educacional na qual a participação dos alunos é o fator de sucesso no aprendizado individual e do grupo, uma vez que é comum fazer-se uso de brainstorm como forma de resolver problemas (BRADSHAW MJ e HULTQUIST BL, 2016).

A gamificação se mostra como uma forma de entretenimento bastante popular e poderosa ferramenta para potencializar aprendizagens. Apesar de ser um termo recente, vem sendo considerado um dos melhores recursos a ser aplicado no processo de ensino e aprendizagem, proporcionando grandes mudanças e facilitando a interação entre a teoria e a prática por meio do lúdico (STOTT $\mathrm{A}$ e NEUSTAEDTER C, 2013).

A gamificação torna as aulas mais atraentes, produtivas, e contextualizadas de forma a manter os estudantes mais participativos e tomadores de decisões, usando recursos próprios através de smartphones, internet própria ou os recursos disponíveis para resolução de situações-problemas (TOLOMEI BV, 2017). 
Diante do exposto, os alunos finalistas, juntamente com o professor da disciplina de saúde da mulher, desenvolveram e aplicaram um jogo didático em hipermídia denominado "Jogo da Reprodução", como recurso metodológico complementar que objetivou promover o entendimento de conteúdos relacionados a infertilidade, métodos contraceptivos, aborto e menopausa a graduandos de enfermagem. A estratégia da utilização do game visou o aprendizado significativo, por meio da indagação, interação com os pares, reflexão, análise e argumentação.

\section{RELATO DE EXPERIÊNCIA}

Trata-se de um estudo descritivo, tipo relato de experiência, elaborado no contexto da disciplina de Trabalho de Conclusão de Curso, ministrada no nono período do curso de Graduação em Enfermagem de uma Instituição de Ensino Superior privada. O planejamento e a realização da sequência didática envolveram seis alunos, os quais são finalistas do curso de Enfermagem, em duas turmas do quinto período do curso e instituição supracitados.

O quinto período foi escolhido observando-se a grade curricular do curso, uma vez que consta a disciplina de Saúde da Mulher. Identificamos por Turma 1 e Turma 2 os locais de desenvolvimento das atividades, que foram acompanhadas pelo professor orientador do estudo e regente das turmas. $\mathrm{Na}$ primeira turma participaram 31 alunos e na segunda turma participaram 30 alunos.

A aula foi planejada com os seguintes objetivos de aprendizagem: Descrever as preocupações comuns relacionadas com a reprodução em termos de sintomas; Identificar os fatores de risco e delinear a orientação apropriada à cliente nos transtornos comuns a reprodução; Comparar e contrastar os diversos métodos anticoncepcionais existentes e sua eficácia geral; Discutir os aspectos fisiológicos e psicológicos da menopausa.

A sequência didática usada foi composta por duas etapas, uma teórica e outra prática usando a hipermídia desenvolvida no formato de game. A aula teórica sobre "Questões comuns relacionadas com a reprodução" foi ministrada na forma expositiva dialogada e desenvolvida com o intuito de identificar o conhecimento prévio dos alunos sobre a temática abordada, que foi debatida e correlacionada ao saber científico.

O conteúdo foi apresentado utilizando-se o programa Microsoft PowerPoint contento textos claros e objetivos, imagens e vídeos, com o intuído de ser agradável e manter a atenção e o interesse dos alunos. A elaboração do conteúdo seguiu as diretrizes da grade curricular do curso e do plano de ensino da disciplina, os quais contemplam a primeira parte da ginecologia.

A segunda etapa se constituiu da aplicação da hipermídia "Jogo da Reprodução", desenvolvido pelos alunos finalistas e professor regente da disciplina, sobre a temática abordada na aula teórica. $O$ jogo foi desenvolvido no formato hipermídia, sendo utilizado o programa Microsoft PowerPoint 2019 para construção do tabuleiro com 28 casas que corresponde ao ciclo menstrual e do quadro com 70 perguntas. Um dado de seis faces foi confeccionado manualmente para determinar a ordem do jogo e o número de casa a avançar no tabuleiro (Figuras 1 e 2). 
Figura 1 - Tabuleiro do Jogo da Reprodução. Manaus-AM, 2020.

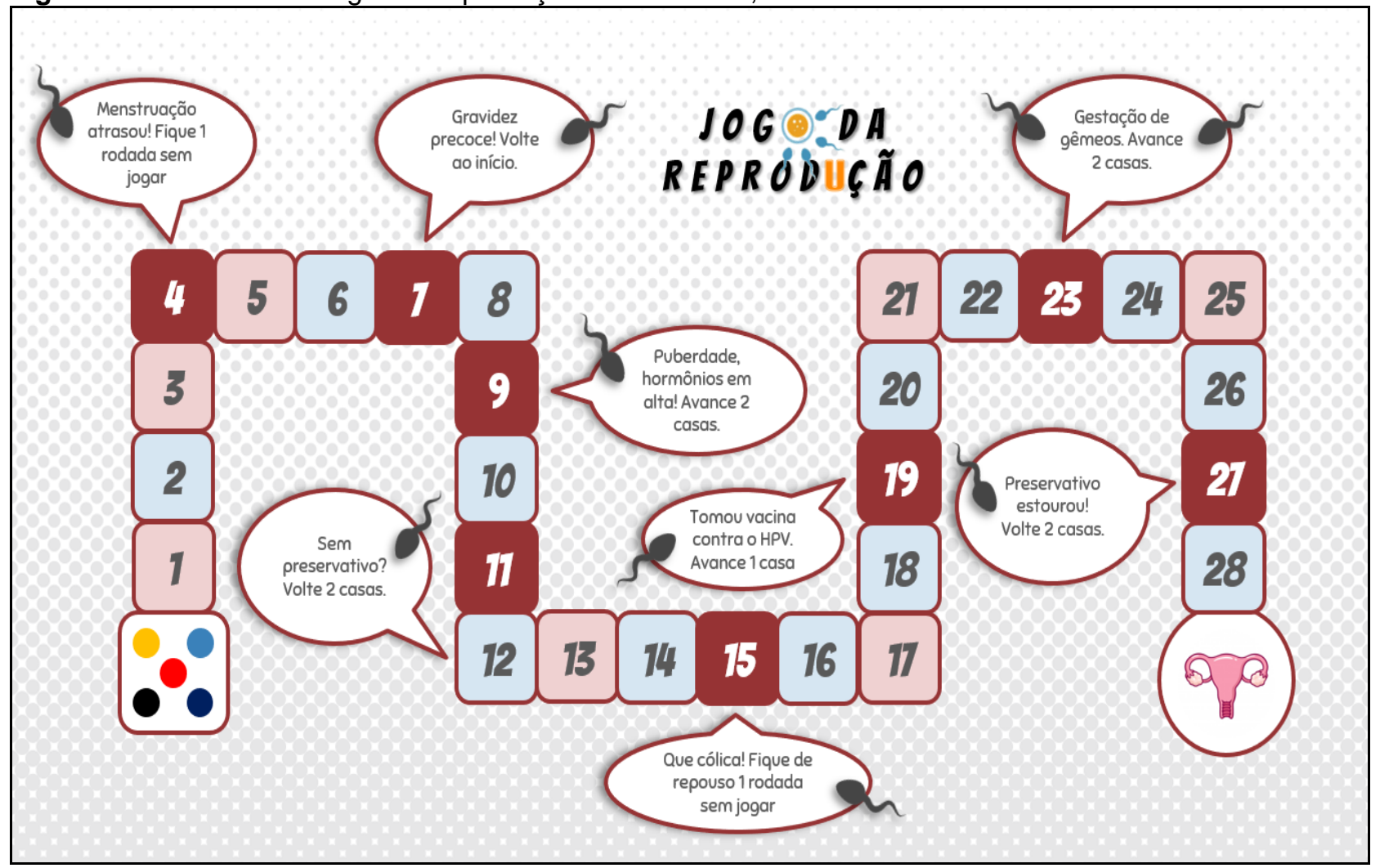

Fonte: BARROS FRB, et al., 2020.

Figura 2 - Quadro de perguntas do Jogo da Reprodução. Manaus-AM, 2020.

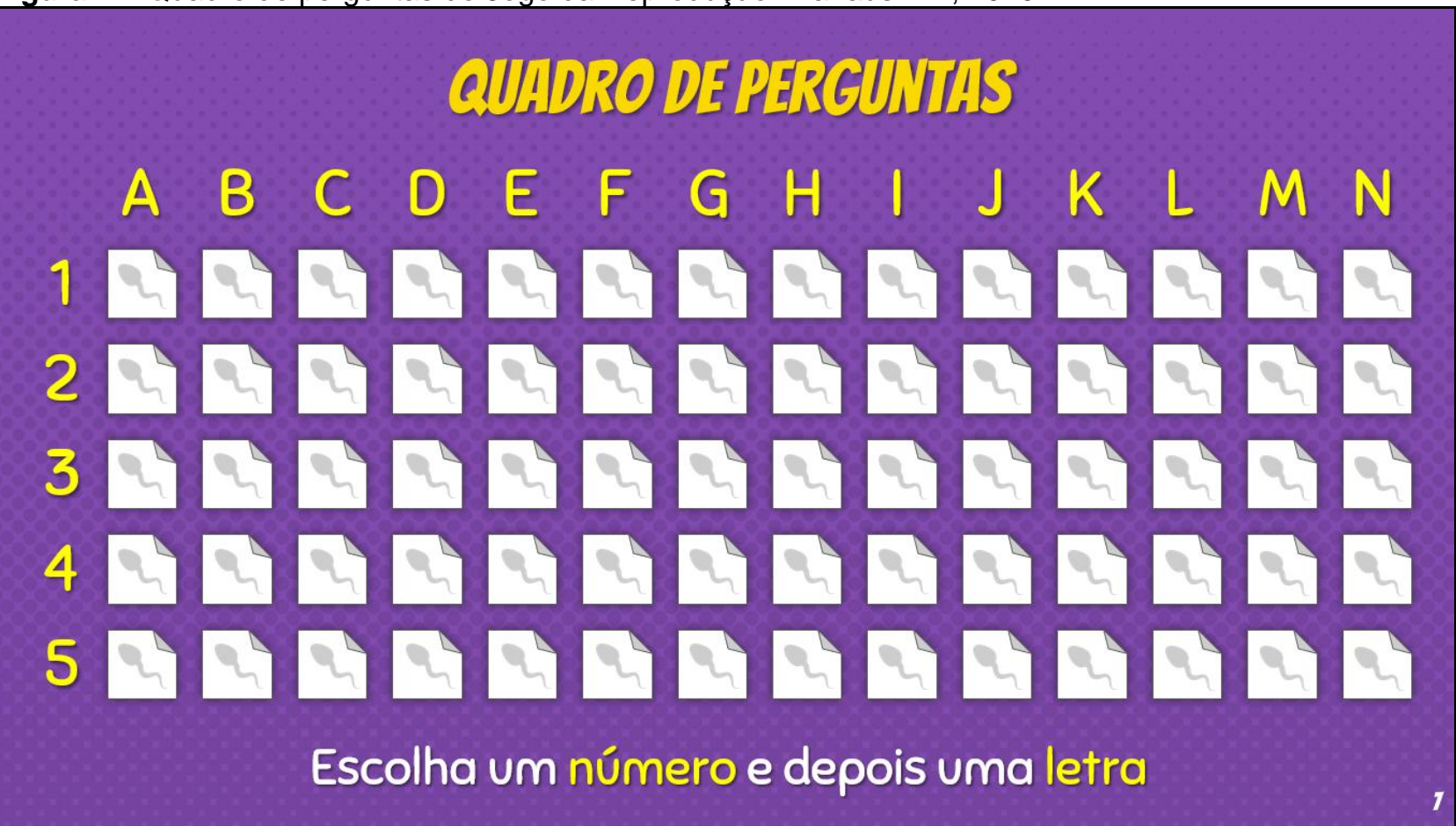

Fonte: BARROS FRB, et al., 2020.

Antes de iniciar o jogo, a turma fora dividida em no máximo cinco grupos, nome e líder foram escolhidos por cada um dos mesmos e as regras foram estabelecidas. Para iniciar o jogo, cada líder deve lançar o dado com a finalidade de determinar a ordem de participação. O líder que tirar o maior número inicia o jogo 
e será seguida a ordem decrescente, onde par ou ímpar será utilizado nos casos de empate. Um quadro de 70 perguntas será apresentado aos grupos, o primeiro líder escolhe número e letra e a pergunta será lida para a primeira equipe, que em caso de acerto, ganha o direito de lançar o dado e prosseguir o número de casas correspondente ao numeral obtido, passando a vez ao grupo seguinte.

Caso a resposta seja incorreta, a equipe perde o direito de lançar o dado, o peão permanece no lugar e a equipe seguinte lança o dado. Quando o pino parar em uma casa surpresa, a equipe deverá seguir as instruções nela apresentada. Vence o jogo a equipe que primeiro atingir a final da trilha (casa 28), para isso, basta obter, no lançamento do dado, um valor igual ou superior ao necessário.

\section{DISCUSSÃO}

$\mathrm{Na}$ aula expositiva, primeira etapa da sequência didática, foram contempladas temáticas sobre os principais distúrbios menstruais, infertilidade, contracepção, aborto, menopausa e condutas de enfermagem, onde muitas concepções foram apresentadas pelos alunos. Mediante a apresentação pôde-se observar maior participação do público feminino, onde muitas das vezes manifestavam suas dúvidas, isso por serem elas as maiores prejudicadas, podendo evoluir para problemas de saúde com sequelas sociais como resultado.

Durante a primeira etapa, fora incentivado um diálogo aberto com a finalidade de conhecer a percepção dos alunos sobre todos os assuntos abordados, levando em consideração os diferentes contextos e saberes entre seus pares. Desta forma, observou-se que a maior parte das inquietações manifestadas era embasada em saberes populares, mitos conhecidos entre a comunidade adolescente e desconhecimento sobre o próprio corpo. Quando questionados sobre os meios de obtenção de esclarecimentos das dúvidas, muitos relataram que recorreram aos meios digitais, principalmente as interações mantidas nas redes sociais, onde a maioria se mostrava incongruente com a literatura científica.

Essa troca de saberes foi de grande importância para a desconstrução de informações erradas extraídas da rede, uma vez que o acesso a informações de saúde na internet apresenta-se como uma questão de saúde pública, não se tratando de discutir a censura ao que é publicado nas mais diversas plataformas digitais, mas sim de garantir que o conteúdo disponibilizado seja verídico e insuspeito, proporcionando segurança ao usuário (MORETTI FA, et al., 2012).

Se tratando especificamente dos métodos de contracepção, notou-se que alguns métodos eram desconhecidos entre os alunos, onde enfatizaram a camisinha e a pílula de contracepção de emergência como os principais métodos conhecidos e que o primeiro, além de prevenir uma gravidez indesejada, também têm uma ótima aceitação na prevenção de Infecções Sexualmente Transmissíveis (IST's), com destaque para o Human Immunodeficiecy Viruses (HIV).

Assimilar as dificuldades que os alunos apresentaram sobre os métodos contraceptivos pôde facilitar o diálogo, compreensão dos demais métodos e as consequências desencadeadas por uso incorreto e/ou discriminado, principalmente sobre o uso de contracepção de emergência. Tais dificuldades podem guardar estreita relação com a percepção sociocultural sobre sexualidade, vulnerabilidade feminina à dominância masculina, a existência ou não de projetos profissionais educativos e informativos, dentre outros (OLSEN JM, et al., 2018).

Debatido todos os temas da aula expositiva e esclarecidas as indagações surgidas, prosseguiu-se para segunda etapa da sequência didática, aplicação do Jogo da Reprodução, onde os alunos demostraram ânimo e participação ativa na solução das questões. Observou-se um esforço coletivo entre os integrantes de cada grupo para ganhar o jogo, assimilação das informações que foram apresentadas, assim como a desconstrução de informações erradas extraídas da internet e/ou de outras fontes.

Por intermédio das respostas obtidas durante o jogo, ficou evidente a absorção de muitas informações adquiridas na aula expositiva (ARAÚJO I, 2016), a desconstrução de informações erradas e a formulação de práticas que poderão auxiliá-los enquanto futuros enfermeiros e enfermeiras no contexto da saúde da mulher, especificamente no que diz respeito a fase do ciclo reprodutivo. 
A utilização de metodologias ativas usadas na prática de ensino e aprendizagem na graduação de enfermagem configura-se como um conceito em evolução, tendo por finalidade ampliar a compreensão dos alunos, voltando sua atenção para um aprendizado embasado nas tecnologias manipuladas por eles (SOUSA MEM, et al., 2018). Dessa forma, possibilita aos graduandos uma prévia da realidade do contexto da prática profissional, preparando-os para novas maneiras de responder aos problemas de saúde e no desenvolvimento de habilidades e competências (SOUZA EFD, et al., 2018).

A gamificação se mostra como relevante metodologia ativa na prática de ensino, uma vez que facilita o aprendizado e a compreensão da temática abordada na forma lúdica, motivacional e interativa, possibilitando uma restrita relação dos conteúdos assimilados com a futura vida profissional, tornando os graduandos mais competentes na argumentação de respostas (SOARES TCS, et al., 2019).

Brum MG e Cruz MEJK (2017), relatam a experiência do uso da gamificação no ensino-aprendizagem de robótica, no qual foi possível constatar uma melhora significativa no desempenho das turmas. O que também é evidenciado no estudo de Tomisaki S, et al. (2016), onde a experiência de gamificação no ensino de gestão de projetos utilizando um jogo de cartas teve resultado positivo, uma vez que motivou os estudantes e melhorou o desempenho acadêmico de maneira lúdica e eficaz. Além da motivação extrínseca, a gamificação permite também a motivação intrínseca, apresentando a análise da relação entre a motivação e o efeito na aprendizagem (POFFO M, et al., 2017).

O objetivo geral do trabalho que era obter um maior engajamento da turma foi atingido, uma vez que os estudantes mantiveram a atenção durante a aplicação da gamificação, pois eles viram a necessidade de aprender, para terem um bom desempenho e, consequentemente, na competição. Além disso, foi comprovado na análise quantitativa um maior desempenho utilizando as metodologias ativas, comparando com as metodologias tradicionais, sendo possível constatar como uma metodologia de ensino impacta no processo de ensino-aprendizagem, através da análise qualitativa e quantitativa.

Portanto, ao compararmos os resultados obtidos com está experiência aos resultados de outros estudos, evidenciamos a relevância do jogo desenvolvido para a identificação dos saberes dos alunos e os problemas conceituais e de compreensão sobre a temática abordada. Dessa forma, o jogo pôde proporcionar aos alunos um ambiente agradável de ensino e aprendizagem participativa, colocando-os no papel de protagonista desse processo, uma vez que os objetivos da atividade foram alcançados (DAYBLACK C, et al., 2015).

\section{CONSIDERAÇÕES FINAIS}

Ao utilizar a técnica de gamificação, os estudantes vivenciaram o aprendizado como um jogo, e com o uso das metodologias ativas, tornando-se protagonista do seu processo de aprendizagem e desenvolveram maior autonomia para explorar novos conhecimentos. Com isso, o aluno deixa de ser um elemento passivo, para ser também gerador de conhecimento. Conclui-se que a gamificação demostrou ser uma ferramenta didática eficaz no apoio as diversas temáticas constantes na grade curricular de graduandos de Enfermagem.

\section{AGRADECIMENTOS E FINANCIAMENTO}

À Coordenação de Enfermagem do Centro Universitário do Norte-UNINORTE/Ser Educacional.

\section{REFERÊNCIAS}

1. ARAÚJO I. Gamification: metodologia para envolver e motivar alunos no processo de aprendizagem. Education in the Knowledge Society, 2016; 17(1): 87-107.

2. AYRES MAC, et al. Influência e inclusão das tecnologias da informação no processo ensinoaprendizagem. Dissertação (Mestrado em Planejamento e Desenvolvimento Regional) - Departamento de Gestão e Negócios - GEN. Universidade de Taubaté, São Paulo, 2014; 171 p. 
3. BRADSHAW MJ, HULTQUIST BL. Innovative teaching strategies in nursing and related health professions. 7 th ed. Burlington, MA: Jones \& Bartlett Learning, 2016; 498 p.

4. BRASIL. Ministério da Saúde. Conselho Nacional de Saúde. Resolução oㅡ 573/2018. Reafirma a prerrogativa constitucional do SUS em ordenar a formação dos (as) trabalhadores (as) da área da saúde e aprova os pressupostos, princípios e diretrizes comuns para a graduação na área da saúde e dá outras providências. Brasília: Ministério da Saúde, 2016.

5. BRUM MG, CRUZ MEJK. Gamificação para o Ensino de Computação na Educação Básica. In: Anais do XXV Workshop sobre Educação em Computação e XXXVII Congresso da Sociedade Brasileira de Computação (SBC), 2017: 2100-2109.

6. DAY-BLACK C, et al. Gamification: An Innovative Teaching-Learning Strategy for the Digital Nursing Students in a Community Health Nursing Course. ABNF Journal, 2015; 26 (4): 90-95.

7. FABBRO MRC, et al. Estratégias ativas de ensino e aprendizagem: percepções de estudantes de enfermagem. Revista Mineira de Enfermagem, 2018; (22): e-1138.

8. KAPP, KM. The gamification of learning and instruction: game-based methods and strategies for training and education. 1st ed. San Francisco: John Wiley \& Sons, 2012: $302 \mathrm{p}$.

9. MORETTI FA et al. Acesso a informações de saúde na internet: uma questão de saúde pública? Revista da Associação Médica Brasileira, 2012; 58(6): 650-658.

10. OLSEN JM, et al. Práticas contraceptivas de mulheres jovens: inquérito domiciliar no Município de São Paulo, Brasil. Revista da Associação Médica Brasileira, 2018; 58(6): 650-658.

11. POFFO M, et al. Gamificaçao: agente motivador na aprendizagem de engenharia de software. In Anais do Computer on the Beach, 2017: 110-119.

12. SOARES TCS, et al. A utilização da gamificação como estratégias de aprendizagem aos alunos de graduação em enfermagem da disciplina SAE III. Ciência Atual-Revista Científica Multidisciplinar do Centro Universitário São José, 2019; 13(1): 02-09.

13. SOUSA MEM, et al. Metodologias ativas no processo ensino-aprendizagem: construção de jogo educativo abordando a assistência de enfermagem ao puerpério. Rev. Saúde Digi. Tec. Edu., 2018; 3 (especial): 62-69.

14. SOUZA EFD, et al. Metodologias ativas na graduação em enfermagem: um enfoque na atenção ao idoso. Revista Brasileira de Enfermagem, 2018; 71(2): 976-980.

15. STOTT A, NEUSTAEDTER C. Analysis of Gamification in Education. School of Interactive Arts and Technology, Simon Fraser University Surrey, BC, 2013; 8 (36): 01-08.

16. TOMISAKI S, et al. MEGA GP: Aplicando a Gamificação no Ensino de Gerência de Projetos. In: Anais do XXIV Workshop sobre Educação em Computação. SBC, 2020: 230-239.

17. TOLOMEI BV. A gamificação como estratégia de engajamento e motivação na educação. EAD em foco, 2017; 7(2): 145-156. 